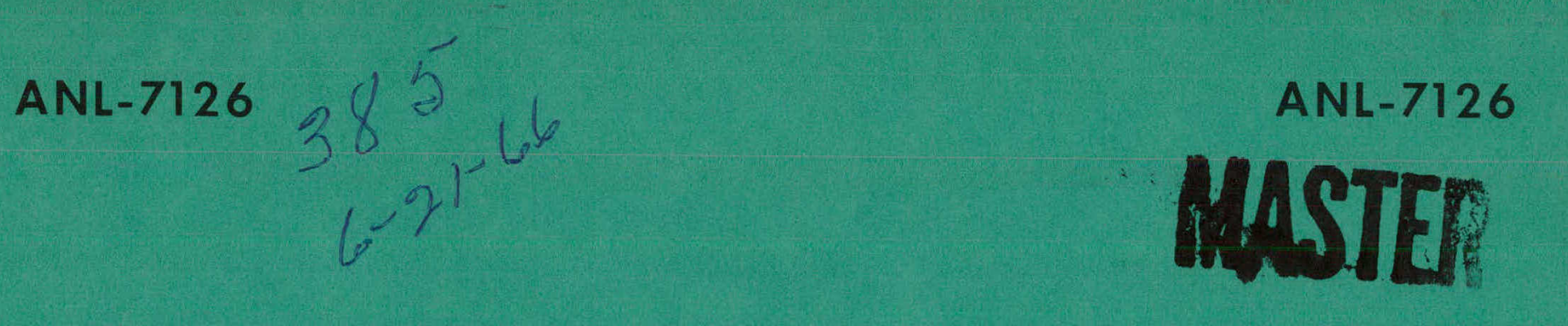

Argonne Rational Laboratory

THE NUMERICAL INTEGRATION OF ORDINARY DIFFERENTIAL EQUATIONS OF VARIOUS ORDERS

by

C. W. Gear

RELEASED FOR AMNOUNCENANI

IN NUCLEAR SCIENCE ABSTRACTS 


\section{DISCLAIMER}

This report was prepared as an account of work sponsored by an agency of the United States Government. Neither the United States Government nor any agency Thereof, nor any of their employees, makes any warranty, express or implied, or assumes any legal liability or responsibility for the accuracy, completeness, or usefulness of any information, apparatus, product, or process disclosed, or represents that its use would not infringe privately owned rights. Reference herein to any specific commercial product, process, or service by trade name, trademark, manufacturer, or otherwise does not necessarily constitute or imply its endorsement, recommendation, or favoring by the United States Government or any agency thereof. The views and opinions of authors expressed herein do not necessarily state or reflect those of the United States Government or any agency thereof. 


\section{DISCLAIMER}

Portions of this document may be illegible in electronic image products. Images are produced from the best available original document. 


\section{LEGAL NOTICE}

This report was prepared as an account of Government sponsored work. Nelther the United States, nor the Lommission, nor any person acting on behalf of the Commission:

A. Makes any warranty or representation, expressed or implied, with respect to the accuracy, completeness, or usefulness of the information contained in this report, or that the use of any information, apparatus, method, or process disclosed in this report may not infringe privately owned rights; or

B. Assumes any liabilities with respect to the use of, or for damages resulting from the use of any information, apparatus, method, or process disclosed in this report.

As used in the above, "person acting on behalf of the Commission" includes any employee or contractor of the Commission, or employee of such contractor, to the extent that such employee or contractor of the Commission, or employee of such contractor prepares, disseminates, or provides access to, any information pursuant to his employment or contract with the Commission, or his employment with such contractor.

Printed in USA. Price $\$ 2.00$. Available from the Clearinghouse for Federal Scientific and Technical Information, National Bureau of Standards

U. S. Department of Commerce, Springfield, Virginia 22151 


\section{ARGONNE NATIONAL LABORATORY 9700 South Cass Avenue Argonne, Illinois 60439}

\section{CFSTI PRICES}

Hi.c. \$2.00: $\mathrm{MN} \cdot 50$

\section{THE NUMERICAL INTEGRATION OF ORDINARY DIFFERENTIAL EQUATIONS \\ OF VARIOUS ORDERS}

by

C. W. Gear

Applied Mathematics Division

RELEASED FOR ANNOUNCEMENT

IN NUCLEAR SCIENCE ABSTRACTS

\section{LEGAL NOTICE}

Thls report was prepared as an account of Government sponsored work. Nelther the Unlted States, nor the Commission, nor any person acting on behalf of the Commission:

A. Makes any warranty or representation, expressed or Implled, with respect to the sccuA. Makes any warranty or representation, expressed or implied, with respect to the sccu-
racy, completeness, or usefulness of the Information contained in thla report, or that the use of any information, apparatus, method, or process diaclosed in this report may not infringe of any Information, apparat
privately owned rights; or

B. Assumes any llabilities with respect to the use of, or for damages resulting from the use of any information, apparatus, mothod, or process disclosed in this report.

As used in the above, "person acting on behalf of the Commission" includes any employee or contractor of the commlesion, or employee of such contractor, to the extent that such employee or contractor of the Commiselon, or employee of such contractor prepares, disseminates, or provides access to, any informatlon pursuant to his employment or contract with the Commission, or his employment with such contractor.

January 1966

Operated by The University of Chicago

under

Contract W-31-109-eng-38

with the

U. S. Atomic Energy Commission 


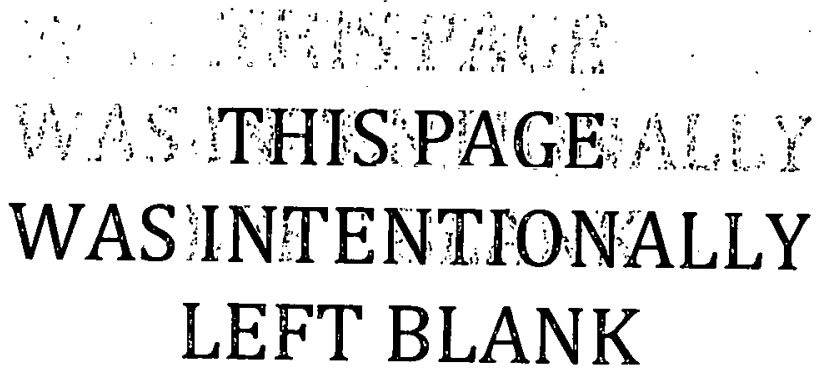


TABLE OF CONTENTS

$\underline{\text { Page }}$

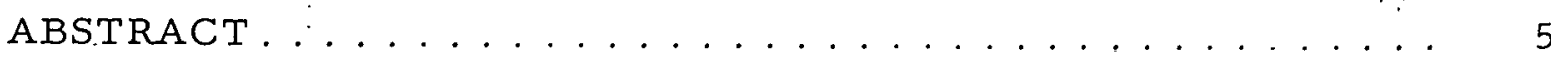

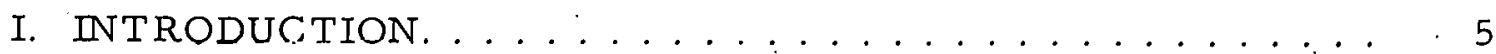

II. STABILIZED MULTISTEP METHOD . . . . . . . . . 7

III. EQUIVALENT: METHODS. . . . . . . . . . . . . . 11

IV. COMPUTA'IIONAL CONSIDERATIONS ......... 14

V. EQUATIONS OF HIGHER ORDER ............ 17

VI. THE CHOICE OF $\ell$ FOR GIVEN EIGENVALUES AND OPTIMUM DEGREE. . . . . . . . . . . 21

VII. CONVERGENCE AND ASYMPTOTIC ORDER OF

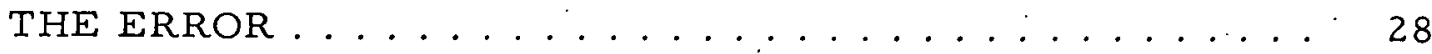

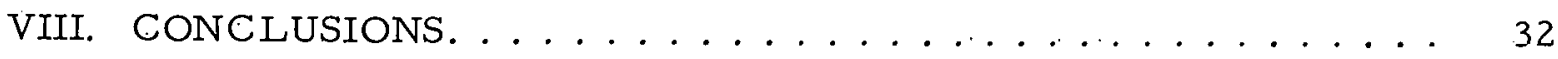
APPENDIXES

A. Modified Predictor-Corrector Methods for First-order Equations with Nonprincipal Roots All Zero. for

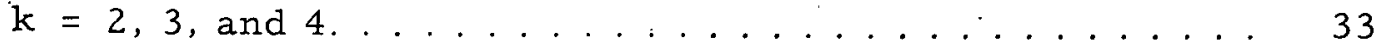

B. Nordsieck's Methods: (N Method with Zero Nonprincipal: Eigenvalues) for $\mathrm{k}=2(1) 8 \ldots \ldots \ldots \ldots$

C. Nordsieck's Methods for $\mathrm{p}=2(1) 4$ and Various $\mathrm{k} \leq 8$ with Zero Nonprincipal Eigenvalues............. 35

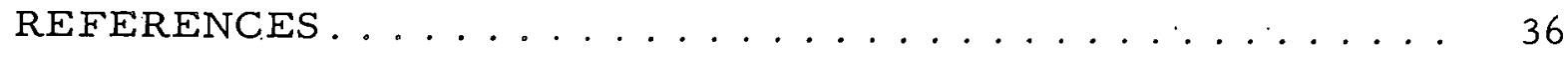




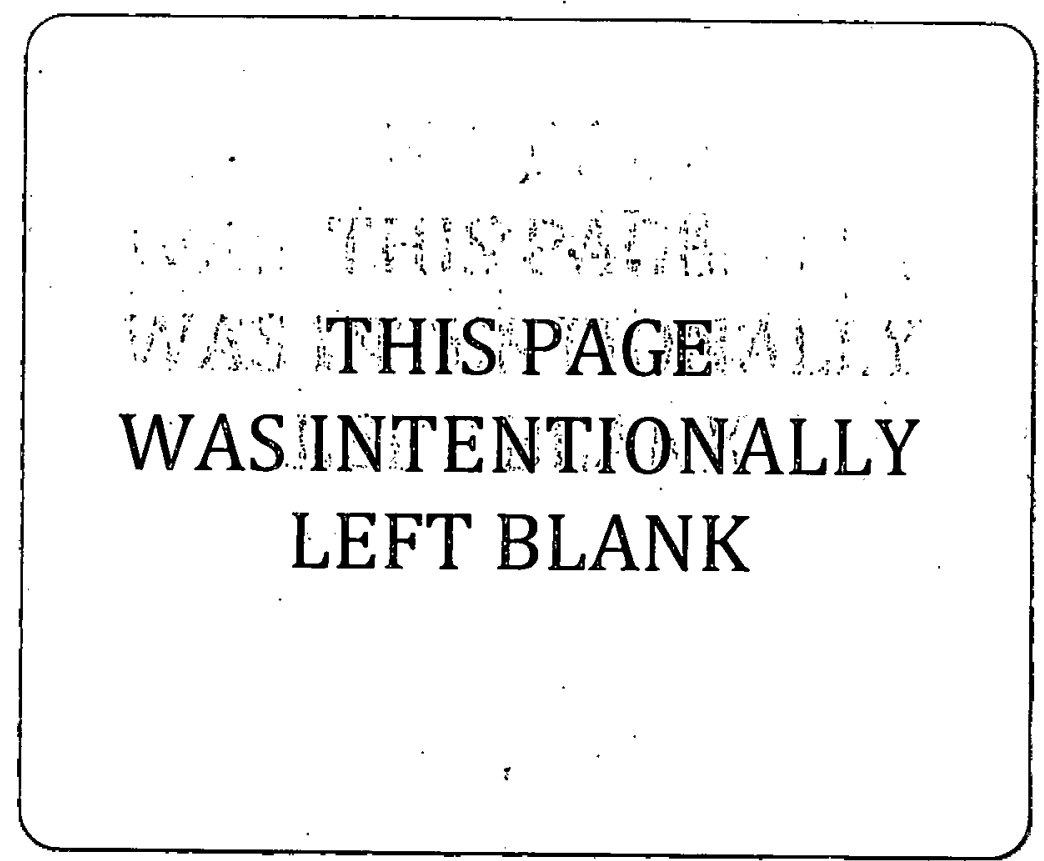




\title{
THE NUMERICAL INTEGRATION OF \\ ORDINARY DIFFERENTIAL EQUATIONS \\ OF VARIOUS ORDERS
}

by

C. W. Gear

\begin{abstract}
This report discusses modified multistep predictorcorrector methods for general initial-value problems and introduces new methods. These new methods are of degree $2 \mathrm{k}$ for $\mathrm{k}$-step methods and require only one function evaluation. Equivalence classes of methods are discussed, and it is argued that the Nordsieck formulation is the best for high-accuracy problems, and that for low-accuracy problems either the Nordsieck formulation or versions of the new methods should be used. The direct application of the methods to higher-order equations is analyzed, and some numerical evidence is given to suggest that this application lcads to faster and more accurate methods than the usual method of integrating a system of first-order equations. Some preliminarytests on the integration of singular families are mentioned. Finally, existence and convergence proofs are presented.
\end{abstract}

\section{INTRODUCTION}

The familiar Dahlquist ${ }^{2}$ result limits the degree of a k-step method to $k+1$ for first-order equations if the method is to be strongly stable. Within this framework, Nordsieck ${ }^{9}$ recast Adams' method into a form suitable for step-size changing. It has been pointed out that changing the step size introduces errors of a lower: degree than those of the regular steps (see Descloux ${ }^{3}$ ), and that step changing is the exception rather than the rule. Some feel that the Nordsieck method requires more work per step, and that there is therefore no reason to prefer it over the Adams method.

This investigation started with a desire to deal with higher-order equations directly (particularly with those representing singular families), rather than by.converting them to a larger system of first-order equations. It was soon realized that the Nordsieck-type methods (to be referred to as the $\mathrm{N}$ methods) were the most applicable to higher-order equations since the derivatives are directly available. In pursuing the relation between 
multistep methods ( $M$ methods) and $N$ methods, we found that when all the methods were formulated after a manner suggested by Descloux, ${ }^{3}$ a larger class of $M$ methods became apparent. These methods have the property that, although they are almost identical to the methods considered by Dahlquist, ${ }^{2}$ they can have degree $2 \mathrm{k}$, be strongly stable, and yet only use one derivative evaluation (although more may be desirable for accuracy reasons).

An equivalence relation between methods is exploited. Under a linear transformation, several $\mathrm{M}$ methods and an $\mathrm{N}$ method (plus any number of mixtures) are equivalent, in that, in the absence of round-off errors, equivalent starting values lead to equivalent answers. A rational decision can therefore be made between equivalent methods on the basis of the roundoff errors and the amount of work involved. It is argued in sectiun IV lial, for high accuracy, the $N$ methods require less work than equivalent melhuds, and that for low accuracy and fixed step size, the new $M$ methods discussed in Section II are marginally better. Almost never, it is suggested, should the conventional Adams multistep methods be used.

The system of equations to be studied consists of $s$ equations in the $s$ unknowns $y_{i}$, their first $p_{i}$ derivatives, and the independent variable $x$. The most general case for which concrete results are given is when these equations can be solved for the highest-order derivatives of each yi so that the equations take the form

$$
F_{i} \equiv\left(y_{i}^{\left(p_{i}\right)}-f_{i}\left(y_{1}^{\left(p_{1}-1\right)}, \ldots, y_{1}, y_{2}^{\left(p_{2}-1\right)}, \ldots, y_{2}, \ldots, y_{s}, x\right)\right) h^{p_{i} /\left(p_{i}\right) !=0}
$$

For didactic reasons, Sections II, III, and IV will deal with the simplest case $s=p_{1}=1$ in order to prepare for a discussion of the advantages of various formulations. Then, in Section $V$, the $N$ methods will be applied directly to Equations (1.1). A numerical comparison of the results of integrating the second-order equation describing $\mathrm{J}_{16}(\mathrm{x})$ directly and integrating the related pair of first-order equations is reported. This preliminary evidence indicates that there is both a time and accuracy advantage to the direct approach. Alșo, some numerical experiments on equations representing singular families that cannot be expressed as (1.1) with Lipschitzian. $f$ are described, although no theoretical results are known.

The standard existence and convergence theorems are left to. Sections VI and VII. The matrix formulation used provides a convergence proof that is equivalent to the usual proof (e.g., Henrici. ${ }^{8}$ ), but is considerably more compact and applies to the system (1.1).

The term "degree" has been used above without definition. To avoid repeated qualification of the word "o'rder," the following three terms are used throughout this report: "Order" is used in reference to a method to 
Miean the asymptotic form $\mathrm{h}^{\mathrm{k}}$ of the error at the end of a fixed finite range of integration as $h \rightarrow 0$. In reference to an equation, it means the order of the highest derivative in the equation. The "degree" of a method is defined by considering one step of the process starting from exact values. If, as

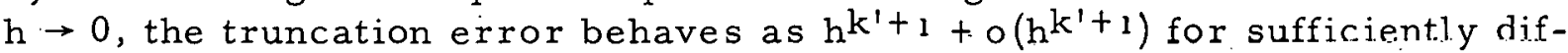
ferentiable functions, then the method has degree $k^{\prime}$. Order is not defined for unstable methods, but stable methods for equations of order $p$, $k$, and $k^{\prime}$ are related by $k=k^{1}+1-p$. The "polynomial degree" of a method is the maximum $k$ " such that for all initial-value problems whose solutions are polynomials of degree $\mathrm{k}^{\prime \prime}$ or less, the method is exact if the starting values are exact. It follows that $k^{\prime \prime} \leq k^{\prime}$.

To reduce the tedium, it will be assumed throughout that all equations and their solutions satisfy suitable Lipschitz and differentiability conditions when needed. They are, in all cases, obvious and well known.

\section{STABILIZED MULTISTEP METHODS}

Consider initial-value problems for the differential equation $y^{\prime}=f(y, x)$ and their numerical solution by means of the general multistep predictorcorrector formulas,

$$
\begin{aligned}
& y_{n+1}^{(0)}=\sum_{j=1}^{k_{1}} \tilde{\alpha}_{j} y_{n-j+1}+h \sum_{j=1}^{k_{2}} \dot{\tilde{\beta}}_{j} f_{n-j+1}, \\
& y_{n+1}^{(m+1)}=\sum_{j=1}^{k_{1}} \alpha_{j} y_{n-j+1}+h \sum_{j=0}^{k_{2}} \beta_{j} f_{n-j+1}^{(m+1)},
\end{aligned}
$$

and

$$
y_{n+1}=y_{n+1}^{(M)}
$$

where

$$
f_{n+1}=f_{n+1}^{(M)}
$$

and

$$
f_{n+1}^{(m)}=f\left(y_{n+1}^{(m-1)}, x_{n+i}\right), \quad m=1,2, \ldots, M
$$

Dahlquist ${ }^{2}$ has shown that the degree of these methods cannot exceed $\max \left[k_{1}, k_{2}\right]+2$ if they are to be stable, and that this degree can only be. 
achieved. if $k_{1}=k_{2}=k$ is even and the method is weakly stable. This process will be modified to enable the degree to be as large as $k_{1}+k_{2}$ while strong stability is retained.

It is convenient to express the numerical process in a matrix notation which is an extension of the notation used by Descloux. ${ }^{3}$ The corrector equation may be written as

$$
y_{n+1}^{(m+1)}=y_{n+1}^{(m)}-\beta_{0}\left(h f_{n+1}^{(m)}-h f_{n+1}^{(m+1)}\right), \quad m=0,1 \ldots, M-1
$$

where

$$
\mathrm{hf}_{\mathrm{n}+1}^{(0)}=\sum_{j=1}^{k_{1}} \frac{\tilde{\alpha}_{j}-\alpha_{j}}{\beta_{0}} y_{n-j+1}+\sum_{j=1}^{k_{2}} \frac{\tilde{\beta}_{j}-\beta_{j}}{\beta_{0}} h_{n-j+1}
$$

Here $h f_{n}^{(0)}$ may be thought of as the predicted value of $h f_{n+l}$. If $F_{n+1}^{(m)}$ is defined as $h f_{n+1}^{(m)}-h f_{n+1}^{(m+1)}$, the iteration of the corrector can be described by

$$
y_{n+1}^{(m+1)}=y_{n+1}^{(m)}-\beta_{0} F_{n+1}^{(m)}
$$

and

$$
h f_{n+1}^{(m+1)}=h f_{n+1}^{(m)}-F_{n+1}^{(m)}
$$

$F_{n+1}^{(m)}$ is the amount by which the latest values of $y$ and $f$ fail to satisfy the differential equation. If the column vector $y_{n}$ is defined as

$$
\underline{y}_{n}^{T}=\left[y_{n}, y_{n-1}, \ldots, y_{n-k_{1}+1}, h f_{n}, h f_{n-1}, \ldots, h f_{n-k_{2}+1}\right]
$$

where $\underline{y}^{T}$ is the transpose of $\underline{y}$, then the numerical process can be expressed as

$$
\begin{aligned}
& \underline{y}_{n+1}^{(0)}=\underline{A}_{n}, \\
& \underline{y}_{n+1}^{(m+1)}=\underline{y}_{n+1}^{(m)}+\underline{l}_{F}\left(\underline{y}_{n+1}^{(m)}, x_{n+1}, h\right),
\end{aligned}
$$

and

$$
\underline{y}_{n+1}=\underline{y}_{n+1}^{(M)}
$$


where $F(\underline{v}, x, h)=v_{k_{1}}-h f\left(v_{0}, x\right)$. (Array elements are indexed from 0.$)$ This will be abbreviated to $F(\underline{v})$, where it is obvious which values of $\dot{x}$ and $h$ are to be used. $A$ is the $\left(k_{1}+k_{2}\right)^{2}$ matrix

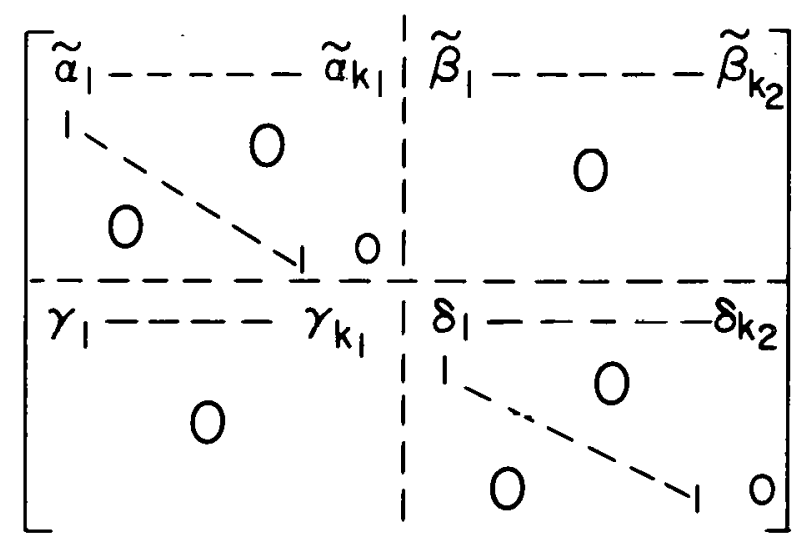

where

$$
\begin{aligned}
& \gamma_{i}=\frac{\tilde{\alpha}_{i}-\alpha_{i}}{\beta_{0}}, \\
& \delta_{i}=\frac{\tilde{\beta}_{i}-\beta_{i}}{\beta_{0}},
\end{aligned}
$$

and $\underline{\ell}^{\mathrm{T}}$ is the vector $\left[-\beta_{0}, 0, \ldots, 0,-1,0, \ldots, 0\right]$.

It has been assumed that the derivative is not re-evaluated after the last corrector application. If the derivative is to be re-evaluated, then one further iteration should be performed by using $\underline{l}=-\underline{e}_{k_{1}}$, where $\underline{e}_{j}$ is the column vector with a one in the $j^{\text {th }}$ position and zeros elsewhere. Feldstein and Stette $r^{4}$ have pointed out that not re-evaluating the derivative can lead to more accurate answers for suitable functions, so it is not certain that such re-evaluation is worthwhile.

An alternative way of viewing this process is as follows: If the process is to be exact for solutions that are polynomials of degree $d$, then a matrix A should be chosen so that (2.3) gives exact values for such polynomials. This equation by itself will not be stable since it takes no account of the differential equation. However, for such solutions, $F\left(y_{n+1}\right)=0$, so that (2.4) defines the same solution in the absence of errors. However, $\underline{\ell}$ can be chosen so as to make the process stable and of higher degree. Consider for a moment the case in which $\partial \mathrm{f} / \partial \mathrm{y}=0$. The method must be stable for this case. Also assume that the solution of the equation $F=0$ is a polynomial of degree $d$ or less so that there are no truncation errors. If the true solution is $\underline{z}_{n}$, define $\underline{\epsilon}_{n}=\underline{y}_{n}-\underline{z}_{n}$. Since $F\left(\underline{z}_{n+1}\right)=0$ and $\underline{z}_{n+1}=A \underline{z}_{n}$, 


$$
\underline{\epsilon}_{\mathrm{n}+1}=\left(I+\underline{\ell} \underline{e}_{\mathrm{k}_{1}}^{\mathrm{T}}\right)^{\mathrm{M}} \mathrm{A} \underline{\epsilon}_{\mathrm{n}}=\mathrm{S} \underline{\epsilon}_{\mathrm{n}} .
$$

The matrix $S$ must therefore be power bounded, that is, all of its roots.must be either inside the unit circle or on the unit circle and simple. In Section VII it is shown that, if $\underline{l}$ is any vector satisfying this condition, and if the degree of the method is at least one, then the method converges.

This leaves the question of what $\underline{\ell}$ to chose. Certainly $\underline{\ell}$ exist for stable methods if the degree is not too large. If the restriction that $\underline{l}$ has only one free parameter $\ell_{0}=-\beta_{0}$ is lifted, then perhaps higher-degree methods can be found. The answer is given in Section VI, namely that if the method has maximum polynomial degree $k_{1}+k_{2}-1$, then an $\underline{l}$ can be found which will make the nonprincipal roots of $S$ take on any desired set of values, and that the degree of the method can be $k_{1}+k_{2}$. 'T'his corresporids to a maximum order predictor and corrector, the latter being one degree higher than the former.

Taking $\mathrm{k}_{1}=\mathrm{k}_{2}=\mathrm{k}$ and choosing $\underline{\ell}$ so that the nonprincipal roots are zero, we obtain an interesting set of stabilized k-step methods. They are of degree $2 \mathrm{k}$, and, with $\mathrm{M}=1$, require only one function evaluation. Previous efforts to get around the Dahlquist stability condition have required two or three function evaluations. (See Butcher, ${ }^{1}$ Gear, ${ }^{6}$ and Gragg and Stetter. ${ }^{7}$ ) In the next section these methods will be shown to be equivalent to the Adams methods on $2 \mathrm{k}$ - 1 points, and also to the Nordsieck methods using $2 \mathrm{k}-1$ derivatives. For $\mathrm{k}=2$, the degree 4 method is

$$
\begin{aligned}
y_{n+1}^{p} & =-4 y_{n}^{c}+5 y_{n-1}^{s}+4 h f_{n}+2 h f_{n-1}, \\
P & =-12 y_{n}^{c}+12 y_{n-1}^{s}+8 h f_{n}+5 h f_{n-1}, \\
f_{n+1} & =f\left(y_{n+1}^{p}, x_{n+1}\right), \\
F & =P-f_{n+1}, \\
y_{n+1}^{c} & =y_{n+1}^{p}-3 F / 8,
\end{aligned}
$$

and

$$
\mathrm{y}_{\mathrm{n}}^{\mathrm{s}}=\mathrm{y}_{\mathrm{n}}^{\mathrm{c}}+\mathrm{F} / 24
$$

The additional step of recorrecting the saved value of $y_{n}$ as well as the predicted value of $y_{n+1}$ is a stabilizing factor. Methods of this type with zero nonprincipal eigenvalues will contain corrections to all the saved values of $y_{n-j}$, but no corrections to the saved values of $f_{n-j}$. However, if nonzero 
nonprincipal eigenvalues are required, then corrections may have to be made to the saved values of $f_{n-j}$. Methods with zero nonprincipal eigenvalues are listed in Appendix A for orders 6 and $8(k=3$ and 4$)$.

In the next section, the $M$ methods discussed above will be shown 1.o be equivalent to $\mathrm{N}$ mcthods and also equivalent to other $\mathrm{M}$ methods with the same value of $k_{1}+k_{2}$. If $k_{2}=1$, the $M$ method is a form of the Adams method (exactly that if the nonprincipal roots are zero). This is usually classified as a $k$-step method, and yet it is equivalent to a $[(k+1) / 2]-s t e p$ method if $k$ is odd. The characteristic that equivalent methods have in common is that they require the same number of items of information about the "history" of the solution, so we will refer to k-value methods where $k$ is llie number of such items.

\section{EQUIVALENT METHODS}

Nordsieck ${ }^{9}$ re-expressed Adams' method in a form convenient for changing the step size, and Descloux ${ }^{3}$ expressed the equivalence of the two methods in terms of a linear transformation. In this section we will summarize these results in order to define the $\mathrm{N}$ methods and equivalent methods and to prepare for a discussion, in Section IV, of their relative merits.

Nordsieck pointed out that, for changing the step size, it is much more convenient to save, as the history of the calculation, the derivatives of the function rather than its values at a number of preceding points. More precisely, he saves scaled derivatives of the approximating polynomial. For calculation, it is slightly more convenient to save the scaled derivatives

$$
a_{j}=\frac{y(j)_{h} j}{j !} \text {. }
$$

Changing the step size by a power of the radix of the computer being used is a fast operation. If $k$ of these values are saved, say $\underline{a}^{T}=\left[a_{0}, a_{1}, \ldots, a_{k-1}\right]$, and if the solution is a polynomial of degree $k-1$, then application of Taylor's series yields

$$
\underline{a}_{n+1}=\underline{A}_{n} \text {, }
$$

where $A$ is the Pascal triangle $A_{i j}=(\underset{j}{j})$, or 


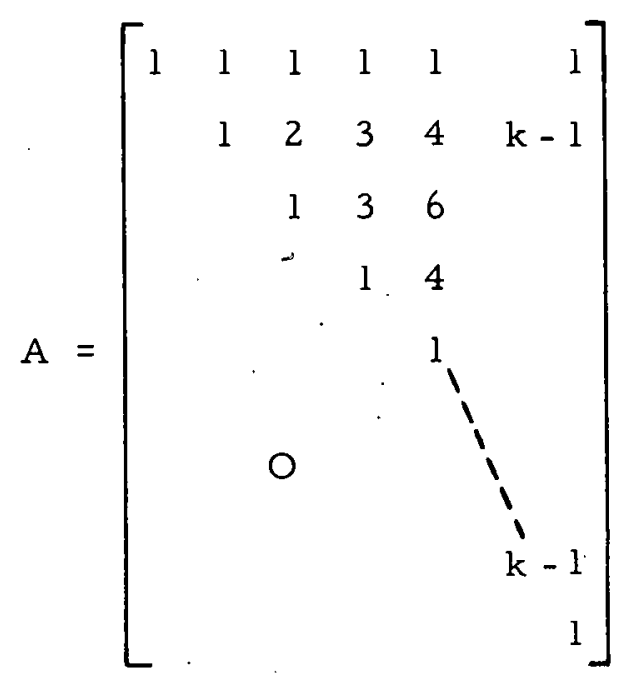

Arguing as before, if the differential equation $F=0$ represents a polynomial of degree $k-1$, then $F\left(\underline{a}_{n}+1\right)$ is 0 , so that a predictor-corrector formula can take the form

$$
\underline{a}_{n}^{(0)} t_{1}=\dot{A}_{\underline{n}} \text {, }
$$

and

$$
\underline{a}_{n+1}^{(m+1)}=\underline{a}_{n+1}^{(m)}+\underline{l} F(\underline{\underline{a} n+1})
$$

Once again, if $M$ corrector iterations are used, $\underline{l}$ must be chosen to make the matrix $S=\left(I+\underline{l} \underline{e}_{1}^{T}\right)^{M}$ A power-bounded.

Suppose now that we are given any $k$-value method in such a matrix form, where the components of the vector $\underline{v}$ of values are supposed to represent linear combinations of the function and its derivatives at various " points. Suppose further that these $k$ combinations form a (not necessarily orthogonal) basis for the space $\mathrm{H}_{\mathrm{k}}$ of polynomials of degree $\mathrm{k}-1$. If any other basis $\underline{b}$ is chosen, and if $\underline{b}=\underline{Q} \underline{v}$, then by a linear transformation of the method

$$
\underline{v}_{n+1}^{(0)}=v_{\underline{n}} \text { and } \underline{v}_{n+1}^{(m+1)}=\underline{v}_{n+1}^{(m)}+\underline{l}_{v} F\left(\begin{array}{c}
(m) \\
v_{n+1}
\end{array}\right)
$$

the method

$$
\underline{b}_{n}^{(0)} f_{1}=B \underline{b}_{n} \text { and } \underline{b}_{n+1}^{(m+1)}=\underline{b}_{n+1}^{(m)}+\underline{l}_{b} G\left(\underline{b}_{n+1}^{(m)}\right) \text {, }
$$

can be obtained, where,

$$
\underline{\mathrm{b}}_{\mathrm{n}}=\mathrm{Qv}_{\mathrm{n}}, \quad \mathrm{B}=\mathrm{QVQ}^{-1}, \quad \underline{\ell}_{\mathrm{b}}=\mathrm{Q} \underline{\mathrm{l}}_{\mathrm{v}}, \quad \text { and } \mathrm{G}(\underline{\mathrm{b}})=\mathrm{F}\left(\mathrm{Q}^{-1} \underline{\mathrm{b}}^{\mathrm{b}}\right) \text {. }
$$


These methods are identical in the absence of rounding error in that if equivalent starting values are chosen, then equivalent answers are obtained.

The class of equivalent methods is therefore just the class of coordinate systems in $\mathrm{H}_{k}$. The $\mathrm{N}$ methods are defined as those obtained by using as a basis for $H_{k}$ the set of scaled derivatives $a_{0}, \ldots, a_{k-1}$. For reference purposes, values of $\underline{\ell}$ for $N$ methods of maximum degree and with zero nonprincipal roots are $\bar{l}$ isted in Appendix B. As an example, the maximum-degree four-value methods with zero nonprincipal roots are given below. The Nordsieck method is

$$
\underline{a}_{n+1}=\left[\begin{array}{cccc}
1 & 1 & 1 & 1 \\
0 & 1 & 2 & 3 \\
0 & 0 & 1 & 3 \\
0 & 0 & 0 & 1
\end{array}\right] \underline{a}_{n}-\left[\begin{array}{l}
3 / 8 \\
1 \\
3 / 4 \\
1 / 6
\end{array}\right] F
$$

If $\underline{z}_{n}=\left[\dot{y}_{n}, . . h \dot{y}_{n}^{\prime}, h y_{n}^{\prime}-1, h y_{n-2}^{\prime}\right]^{T}=Q_{1} \underline{a}_{n}$,

where

$$
Q_{1}=\left[\begin{array}{rrrr}
1 & 0 & 0 & 0 \\
0 & 1 & 0 & 0 \\
0 & 1 & -2 & 3 \\
0 & 1 & -4 & 12
\end{array}\right]
$$

then

$$
\underline{\mathbf{z}}_{\mathrm{n}+1}=\left[\begin{array}{cccc}
1 & 23 / 12 & -4 / 3 & 5 / 12 \\
0 & 3 & -3 & 1 \\
0 & 1 & 0 & 0 \\
0 & 0 & 1 & 0
\end{array}\right] \mathbf{z}_{n}-\left[\begin{array}{c}
3 / 8 \\
1 \\
0 \\
0
\end{array}\right] \mathrm{F}
$$

is the Adams method, and if

$$
\underline{y}_{n}=\left[y_{n}, y_{n-1}, h_{n}^{\prime}, h_{n-1}^{\prime}\right]^{T}=Q_{2} \underline{a}_{n},
$$

where

$$
Q_{2}=\left[\begin{array}{cccc}
1 & 0 & 0 & 0 \\
1 & -1 & 1 & -1 \\
0 & 1 & 0 & 0 \\
0 & 1 & -2 & 3
\end{array}\right],
$$


then

$$
\underline{y}_{n+1}=\left[\begin{array}{rrrr}
-4 & 5 & 4 & 2 \\
1 & 0 & 0 & 0 \\
-12 & 12 & 8 & 5 \\
0 & 0 & 1 & 0
\end{array}\right] \underline{y}_{n}-\left[\begin{array}{c}
3 / 8 \\
-1 / 24 \\
1 \\
0
\end{array}\right] F
$$

is the new $M$ method.

\section{COMPUTATIONAL CONSIDERATIONS}

In Section III, methods were grouped. into equivalence classes such that within a class, the numerical behavior was determined solely by roundoff error. Therefore a rational decision between methods within one class can be made on the basis of this and the number of basic computational operations required. The problem of wich class to use will not be discussed further since.it depends much more heavily on characteristics of the differential equation, such as $\partial f / \partial y$. In general, these characteristics are not fully known.

Nordsieck's motive in picking the scaled-derivative representation was to simplify the step-changing process. Various authors. (e.g., Descloux ${ }^{3}$ ) have commented that step modification is the exception rather than the rule, and that, since the $\mathrm{N}$ methods appear to involve more computational operations per step, perhaps the classical methods are preferable. This section presents some arguments in favor of the $\mathrm{N}$ methods and some in favor of the $M$ methods and suggests. that the Adams and conventional multistep mcthods should almost never be used.

The critical parameter in the choice of method within an equivalence class is the ratio of the word size of the computer used to the desired accuracy. If round-off errors are insignificant at each step of the process as compared to the truncation errors, then all the methods we have discussed can be handled in single precision. If, however, round-off is significant, then some multiple precision work is to be expected.

It is common, in Runge-Kutta integration, to do double-precision accumulation of the dependent variable values while calculating $h f(x, y)$ to single precision. The justification for this is that the scale factor of $h$ reduces the value of $|\mathrm{hf}|$ relative to that of $|y|$. This argument can be extended to the $N$ methods. The numbers being saved are values of $a_{j}=\left(h j_{y}(j) / j !\right)$. Since these are the terms of the Taylor series of $y(x)$, they can be expected to reduce in. relative size as $\mathrm{j}$ increases. Indeed the first neglected term must be small or the truncation error of the method may: be bad! Thus each successive element of a requires successively fewer digits. If high precision is required in $y$, say $m$-word precision, then we may expect to keep 
about $(k-j) m / k$ words of precision for $a_{j}$. In contrast, multistep methods would require each value of $y_{n-j}$ to be saved in full $m$-word precision, and each scaled derivative $\mathrm{hf}_{\mathrm{n}-\mathrm{j}}$ to be saved to slightly less precision, all at a corresponding increase in arithmetic storage.

Some objections have been raised to the approximately $(k-1)(k-2) / 2$ multiplications required in evaluating Aa for $N$ methods as compared to the approximately $2 \mathrm{k}$. required for $M$ methods. Because of the properties of Pascal's triangle, Aa can be evaluated without any multiplications and with no extra additions over the $k(k-1) / 2$ multiplications obviously needed. This is best demonstrated by the following section of ALGOL program which performs one integration step using $M$ corrector iterations for a single differential equation as sumed to be specified by

$$
\text { function }(a, x, h)=a[i]-h * f(x, a[0])=0 \text {. }
$$

\section{PROGRAM}

$$
\begin{aligned}
& \text { for } \mathrm{i}:=0 \text { step } 1 \text { until } \mathrm{k}-2 \text { do } \\
& \text { for } j:=k-1 \text { step }-1 \text { until } i+1 \text { do } \\
& a[j-1]:=a[j-1]+a[j] ; \\
& \mathrm{x}:=\dot{x}+\mathrm{h} \text {; } \\
& \text { for } i:=1 \text { step } 1 \text { until } M \text { do } \\
& \text { begin } f:=\text { function }(a, x, h) \text {; } \\
& \text { for } j:=0 \text { step } 1 \text { until } k-1 \text { do } \\
& a[j]:=a[j]+f * \ell[j] ;
\end{aligned}
$$

end;

For methods with zero nonprincipal roots, $\ell[1]=-1$, so that the multiplication of $\mathrm{f} * \ell[\mathrm{j}]$ need not be performed at each corrector iteration except for $j=0$; thus the number of multiplications in Nordsieck's method need only be $k+M-2$. Another $k(k+1) / 2+2 M-2$ additions are needed in addition to $M$, evaluations of $h f(x, y)$. In contrast, Adams' method requires $2 \mathrm{k}+\mathrm{M}-2$ multiplications and $2 \mathrm{k}+2 \mathrm{M}-3$ additions, while the $M$ methods of Section II require $5 \mathrm{k} / 2+M-1$ multiplications and $5 \mathrm{k} / 2+$ $2 \mathrm{M}-3$ additions. These figures are summarized in Table I. 
TABLE I. Number of Operations Required

\begin{tabular}{|l|l|l|}
\hline Method & Multiplications & Additions \\
\hline Nordsieck & $\mathrm{k}+\mathrm{M}-2$ & $\mathrm{k}(\mathrm{k}+\mathrm{l}) / 2+2 \mathrm{M}-2$ \\
Adams & $2 \mathrm{k}+\mathrm{M}-2$ & $2 \mathrm{k}+2 \mathrm{M}-3$ \\
$\mathrm{M}$ Method & $5 \mathrm{k} / 2+\mathrm{M}-1$ & $5 \mathrm{k} / 2+2 \mathrm{M}-3$ \\
\hline
\end{tabular}

However, this is not quite the full story. In multistep methods, the previous values must be saved, which means that either "clever" indexing and indirect addressing must be used (fairly time-consuming if done in source language for most compilers) or the numbers must be moved in store, which is also time-consuming. If the step size is to be changed, this is particularly bad since it is usual to save double the number of previous values for possible step doubling.

Starting procedures recommended for multistep methods usually involve a number of Runge-Kutta steps, on a smaller step size if a higher than third-degree method is to be used. The space occupied by the additional program is no longer a problem with present-day memories, but the $\mathrm{N}$ methods are amenable to simple starting processes that require little additional program. Nordsieck gives details of one such method. Other methods that use sequences of vectors $\underline{l}$ over sequences of step sizes can readily be devised.

A potential advantage of $\mathrm{N}$ methods that has not yet been exploited is the possibility of adjusting the order of the method according to the behavior of the equation. Many schemes have been devised for adjusting the step size based on the value of the predictor-corrector difference (in this case, just $F$ ), but multistep methods are difficult to organize efficiently if the order is to be changed from time to time. It seems plausible that an analysis of the sizes of the components of a could give some indication of what order to choose.

To summarize, the $\mathrm{N}$ methods are advantageous when higher than single precision is required; in single precision there is not too much difference. If a Runge-Kutta starter is to be used, then the $M$ methods of Section II require fewer points, although at the point.where round-off is first becoming important, the Adams methods might have a slight edge. For a general-purpose routine, this writer recommends an $\mathrm{N}$ method, particularly because, as is to be discussed in the next section, it is directly applicable to higher-order equations. Since many problems that are integrated on a computer are second- or higher-order systems, a considerable saving can be effected by treating them as such, rather than as a larger system of first-order equations. 


\section{EQUATIONS OF HIGHER ORDER}

Suppose we wish to solve an initial value problem for the set of $s$ simultaneous ordinary differential equations

$F_{i}\left(\underline{a}_{1}, \underline{a}_{2}, \ldots, \underline{a}_{s}\right)=\frac{h^{p_{i}}\left(p_{i}\right)}{p_{i} !}-\frac{h_{1} p_{i}}{p_{i} !} f_{i}\left(y\left(p_{1}-1\right), \ldots, y_{1}, y_{2}\left(p_{2}-1\right), y_{2}, \ldots, y_{s}, x\right)=0$,

where

$$
a_{i}=\left[\dot{y}_{i}, h y_{i}, \frac{h^{2}}{2 !} y_{i}^{\prime \prime}, \ldots, \frac{h^{k_{i}-1} y_{i}\left(k_{i}-1\right)}{\left(k_{i}-1\right) !}\right]^{T} \text {. }
$$

The usual technique is to handle these as a system of $\sum_{i} p_{i}$ first-order equations. (If all of the derivatives are absent from the functions $f$, then special methods exist to handle them directly.) Even when this condition is not met, the $N$ methods discussed above are particularly suitable for the dircct integration of these equations since the derivates are already available, apart from a scale factor. Under linear transformations, equivalent methods can be generated, but this section will restrict its attention to the $N$ methods. The results (derived in Sections VI and VII) will be discussed, and a numerical comparison between an integration of $\mathrm{y}^{\prime \prime}+\mathrm{y}^{\prime} / \mathrm{x}+(1-256)$ $\left.\mathrm{x}^{2}\right) \mathrm{y}=0\left(\mathrm{~J}_{16}(\mathrm{x})\right)$ by methods for second-order equations and by methods for a system of first-order equations will be presented. Finally some remarks will be made about more general sets of equations that represent singular families.

To simplify the discussion, $s$ is taken as 1 , and we consider methods of the form

$$
\begin{aligned}
& \underline{a}_{n+1}^{(0)}=\underline{a}_{n}, \\
& \underline{a}_{n+1}^{(m+1)}=\underline{a}_{n+1}^{(m)}+\underline{l} F\left(\underline{a}_{n+1}^{(m)}, x_{n+1}, h\right), \\
& \underline{a}_{n+1}=\underline{a}_{n+1}^{(M)},
\end{aligned}
$$


for the differential equation

$$
F(\underline{a}, x, h)=0=\frac{h^{p}}{p !}\left(y^{(p)}-f\left(y^{(p-1)}, y^{(p-2)}, \ldots, y, x\right)\right) .
$$

If $A$ is the $k \times k$ Pascal matrix, then the method is exact for polynomials of degree $k-l$ or less. If we consider the case where $f$ is independent of $y$ and its derivatives, it is immediate that a necessary condition for stability is that

$$
S=\left(I+\underline{\ell} \underline{e}_{p}^{T}\right)^{M} A
$$

must represent a stable process; that is, its eigenvalues must all either lie inside the unit circle or be not more than $\mathrm{p}$-fold on the unit circle. Indeed, with the usual condition of accuracy or consistency, this condition is. sufficient in the case that $f$ only depends on $y$ and not on its derivatives. From the form of $S$ it is apparent that it always has $p$ eigenvalues at $z=1$. If the highest derivative of $y$ that appears in $f$ is $y(p-q)$, then methods with roots greater than $\mathrm{q}$-fold on the unit circle (except for $\mathrm{z}=1$ ) may give rise to nonconvergent methods. Both convergent and nonconvergent methods can be found if the matrix $A$ is such that the method is not of maximum polynomial order $k$ - 1 . If the method is of maximum order, then it will be nonconvergent. This can readily be seen by considering the case $y(p)=y(p-q)$. This is the same as integrating $y(q)=y$ by a method using the $(k-p+q) \times(k-p+q)$ Pascal-triangle matrix, and then integrating the result $p$ - $q$ times. (Note that the matrix $A_{t}=\left(\begin{array}{l}t+j \\ t+i\end{array}\right)$ is similar to the Pascal matrix $\left(\begin{array}{l}j \\ i\end{array}\right)$ under the transformation diag $\left[d_{i}=\left(\begin{array}{c}t+i \\ i\end{array}\right)\right]$.) In general, it does not seem worth using methods of other than maximum order; it is therefore better to demand that all nonprincipal roots are simple if on the unit circle. Since the reason for choosing roots on the unit circle was to achieve the maximum order permitted under the Dahlquist theorem, which is no longer applicable under the relaxing of the values permitted for $\underline{\ell}$, this represents no real restriction in practice. It is shown in Section VI that, as for firstorder methods, if the method is of maximum polynomial degree, then $\underline{\ell}$ can be chosen to make the nonprincipal roots any desired set of values. This only specifies the last $\mathrm{k}$ - $\mathrm{p}$ elements of $\underline{\ell}$, leaving $\mathrm{p}$ to be determined for optimum accuracy. It is also seen in Section VI that if $q$ is as defined above $(y(p-q)$ is the highest derivative appearing in $f)$, then these remaining elements of $\ell$ can in general be chosen to achieve the limiting accuracy of $\mathrm{k}+\mathrm{q}-\mathrm{l}$ degree. Again, this can be intuitively seen to be reasonable by considering the equation $\mathrm{y}^{(\mathrm{q})}=\mathrm{y}$ and integrating the result $\mathrm{p}-\mathrm{q}$ times.

Convergence of these methods for systems of equations is proved in Section VII under the stability condition above (all nonprincipal roots are inside the unit circle or are simple on the unit circle) and a consistency 
condition that the degree of the method is at least p. That is, if the $N$ methods are used, it is sufficient for consistency that $k \geq p$. Appendix $\dot{C}$ lists the vectois $\underline{\ell}$ for various $\mathrm{p}$ and $k$.

To compare the direct approach numerically, $\mathrm{J}_{16}(\mathrm{x})$ was integrated by methods for $\mathrm{p}=1$ and $\mathrm{p}=2$ with $\mathrm{k}=5,6$, and 7. A fixed step size in the range $x=6(h) E$ was used, where $h=1 / 4,1 / 8$, and $1 / 16$ while $E=6132,6134,6136$, and

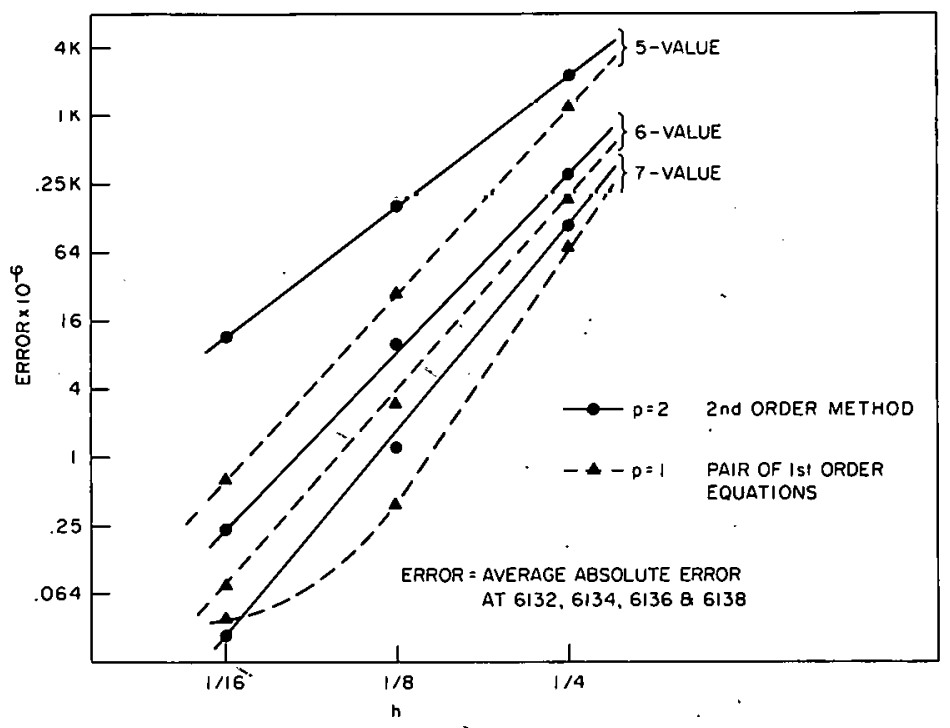

Fig. 1. Results of Integration of $y^{\prime \prime}+y^{1 / x}+\left(1-256 / x^{2}\right) y=0$ for $\mathrm{x}=6(\mathrm{~h}) 6138$
6138 (these are the cases given in Nordsieck's paper ${ }^{9}$ ). In Fig. 1, the average absolute error at the four end points is plotted aydinsl step size for the six methods. Log-log paper is used, so that the slope of the lines (indicated near their centers) should be the order of the method. These calculations were performed in singleprecision floating-point arithmetic in FORTRAN on a CDC-3600, so that roundoff should not be a factor. Starting values for $\mathrm{J}$ and $\mathrm{J}^{\prime}$ were taken from Nordsiecks paper, and a simple-minded

starting procedure was used.in all cases, consisting of setting all other derivatives to zero and then doing eight steps of $\mathrm{h} / 16$, followed by four steps each of $\mathrm{h} / 8, \mathrm{~h} / 4$, and $\mathrm{h} / 2$. The position of the point $\mathrm{h}=1 / 16$ on $\mathrm{p}=1, \mathrm{k}=7$ is believed to be due to starting error, but the strange behavior of the line $\mathrm{p}=1, \mathrm{k}=5$ is not understood. It is probably due to an unlucky choice of end points for measuring the errors (they oscillate in sign over the range).

The order of the methods is $k-p+l$ as shown in Section VII. Comparing $\mathrm{p}=1$ and $\mathrm{p}=2$ methods of the same order, we see from Fig. 1 that there is a factor of two in the error in favor of $p=2$ methods. This is not a significant amount, but the amount of work in the case of $p=2$ is considerably less. About the same amount of work is required for function evaluations, but the $\mathrm{k}$-value method for two equations requires about $k-1+M-2$ multiplications and $(k-2)(k+1) / 2+2 M-1$ additions per step more than the $(k+1)$-value method for one equation. The number of initial values required is the same in either case.

In the case of some systems of equations, some components are very slowly varying and therefore can be treated with a much larger step 
size than that needed for a rapidly varying: component. If the differential equation defined by $F_{i}\left(\underline{a}_{j}\right)$ is complex to evaluate, the algorithm of this report can be adapted to give considerable savings in computation. To take a specific example, suppose that the pair of simultaneous differential equations

$$
F_{1}\left(\underline{a}_{1}, \underline{a}_{2}, x\right)=0 \text {, }
$$

and

$$
F_{2}\left(\underline{a}_{1}, \underline{a}_{2}, x\right)=0 \text {, }
$$

are to be solved and it is known that $\underline{a}_{1}$ is such that $\vec{F}_{1}$ can be treated with a. step size $q$ times larger than $F_{2}$ for $\underline{a}_{2}$. The algorithm consists simply of correcting $\underline{a}_{1}$ by $\underline{\ell}_{1} F_{1}$ only at every $q^{\text {th }}$ step. This means that $q-1$ values of $\underline{a}_{1}$ are obtained by extrapolation from a given point, and then $a$ correction is applied. In the absence of an influence from $\underline{a}_{2}$, this is identical to using a step of size $q$ h. Thus the function evaluations are saved, although it is necessary to perform the extrapolation to each intermediate step.

As a postscript of this section, some comments will be made about more general systems of equations in which the highest derivatives do not appear explicitly. If the matrix

$$
G=\frac{\partial F_{i}}{\partial y_{j}^{\left(p_{j}\right)}}, i, j=1,2, \ldots, s
$$

is nonsingular in the neighborhood of the solution, then for small enough $h$, the $F_{j}$ can be used as multipliers of vectors $\underline{\ell}_{i j}, i, j=1, \ldots, s$, which are dependent on the matrix $G$, and they can be added to the vector $\underline{a}_{i}$, in $a$ method that essentially amounts to solving the equations locally for the $y_{i}\left(p_{i}\right)$. The case of real interest occurs when the matrix $G$ has a singularity in the range of integration. The system of equations has a singularity that may show up as a singularity in the solution, in which case polynomial methods are of no use, or it may show up as a singularity in the family of solutions but not in an individual solution, such as a common point or an envelope. In these cases, polynomial methods should be applicable, but current methods break down. Three examples of singularities at $\mathbf{x}=0$ are given below:

$$
\begin{aligned}
& F_{1}=x y^{\prime}-1=0 ; y=\log (c x) \text {, singularity in the solution. } \\
& F_{2}=x y^{\prime}-y \log (y)=0 ; y=e^{c x} \text {, common point }(0,1) \text { for the family. } \\
& F_{3}=y^{\prime 2}+y^{2}-1=0 ; y=\sin (x+c) \text {, envelope } y= \pm 1 \text { for the } \\
& \text { family. }
\end{aligned}
$$


The last two equations were successfully integrated by means of an $N$ method in which the vector $\underline{\ell}$ was changed according to the.known derivatives of $F$ with respect to $y$ and $y^{\prime}$. In fact, if $\ell_{q}$ is defined as that value of $\underline{\ell}$ such that all nonprincipal roots of $\left(\mathrm{I}-\underline{\ell}_{\mathrm{q}}^{\mathrm{T}}\right) \mathrm{A}$ are zero, then the $\underline{\ell}$ used at each step was given by

$$
\underline{\ell}=\frac{\sum_{q=0}^{p} \underline{\ell}_{q} F_{y}(q)}{\sum_{q=0}^{p} F_{y}^{2}(q)}
$$

(a fairly obvious heuristic choice).

In general, this technique is not practical since $F_{y}(q)$ is not known; neither is a convergence proof known for this case, but the technique does suggest ways in which problems with singularities might be tackled

\section{THE CHOICE OF $\underline{\ell}$ FOR GIVEN EIGENVALUES AND OPTIMUM DEGREE}

In a method for a $\mathrm{p}^{\text {th }}$-order equation, the last $\mathrm{k}$ - $\mathrm{p}$ elements of $\underline{\ell}$ are chosen according to the nonprincipal eigenvalues desired, while the first $p$ elements can be chosen to maximize the degree. Two theorems will be presented in this section. One states that for a k-variable method of polynomial degree $k-1$, the last $k$ - p elements can always be chosen to make the $k$ - p nonprincipal eigenvalues take on any desired set of values. The second states that a method of degree $k$ always exists, and that under further conditions the degree can be increased to $\mathrm{k}+\mathrm{q}-\mathrm{l}$, where $\mathrm{q}$ is the smallest integer $\leq \mathrm{p}$ such that

$$
\frac{\partial f}{\partial y(p-q)} \neq 0
$$

Because any unique representation can be chosen for $\mathrm{H}_{\mathrm{k}}$, we will work with the $\mathrm{N}$ methods in the next two sections. In this representation, methods of polynomial degree $k-1$ will use the Pascal matrix of order $k$ for the predictor formula.

Theorem 1. If $A$ is the $k \times k$ Pascal matrix $A_{i j}=\left(\begin{array}{l}j \\ i\end{array}\right)$ and if $e_{p}^{T}$ is the k-element row vector with a one in the $\mathrm{p}^{\text {th }}$ position (numbering starts from zero). and zeros elsewhere, then for any set of $k-p$ numbers $\left\{\lambda_{i}\right\}$ a column vector $\ell$ exists such that the matrix 


$$
\left(I+\underline{\ell} e_{p}^{T}\right)^{M} \cdot A
$$

has $p$ eigenvalues equal to one and $k$ - $p$ eigenvalues equal to the set $\left\{\lambda_{i}\right\}$. The eigenvalues are independent of the first $p$ elements of $\underline{l}$ and uniquely determine the last $k$ - p elements of $\underline{l}$.

Proof. The independence of the first $p$ elements of $\underline{\ell}$ is obvious from the form of the matrix. Also, this form implies that the first $p$ eigenvalues are 1. Therefore we can take $p=0$, and $A$ to be the $(k-p) x(k-p)$ lower principal minor of $A$. The only properties that are required of $A$ are that it be upper triangular with equal nonzero diagonal elements and nonzero first off-diagonal elements $A_{i, i+i}$. This is true for all principal minors of Pascal matrices. First note that

$$
\left(\mathrm{I}+\underline{\ell}_{\mathrm{e}_{0}}^{\mathrm{T}}\right)^{\mathrm{M}}=\mathrm{I}+\mathrm{c} \underline{\mathrm{l}} \underline{\mathrm{e}}_{0}^{\mathrm{T}}
$$

where

$$
c=\left\{\begin{array}{cc}
\left(\left(1+\ell_{0}\right)^{M}-1\right) / l_{0} & \ell_{0} \neq 0 \\
M & l_{0}=0
\end{array}\right\} .
$$

Hence it is sufficient to consider $M=1$ : Let

$$
\mathrm{T}=\mathrm{A}\left(\mathrm{I}+\stackrel{\ell \mathrm{e}_{0}^{\mathrm{T}}}{\mathrm{T}}\right)
$$

It has the same eigenvalues as

$$
S=\left(I+\underline{e}_{0}^{T}\right) A
$$

$\mathrm{T}=\mathrm{A}+\underline{\mathrm{u}}_{\mathbf{e}_{0}^{\mathrm{T}}}^{\mathrm{T}}$, where $\underline{\mathrm{u}} .=\mathrm{A} \underline{\underline{\ell}} \cdot \mathrm{We}$ will show in the lemma below that $\mathrm{a}$ similarity transformation. $\bar{Q}$ of $A$ exists that puts $A$ in a Jordan form with nonzero elements immediately above the diagonal, and in which $Q$ and $Q^{-1}$ have the triangular form

$$
\left[\begin{array}{ccccccc}
1 & 0 & 0 & 0 & : & \cdots & 0 \\
0 & 1 & \mathrm{x} & \mathrm{x} & \ddots & \ddots & \mathrm{x} \\
0 & 0 & 1 & \mathrm{x} & \ddots & \cdots & \mathrm{x} \\
& \cdots & \cdots & & \cdots & \cdots & \cdot \\
0 & 0 & 0 & 0 & \ddots & \cdot & 1
\end{array}\right],
$$

where $\mathrm{x}$ represents any element. 
It then follows that

$$
Q^{-1} \mathrm{TQ}=Q^{-1} \mathrm{AQ}+\mathrm{Q}^{-1} \underline{u}_{-0}^{\mathrm{T}} \mathrm{T}=\mathrm{Q}^{-1} \mathrm{AQ}+\underline{\mathrm{ve}}_{0}^{\mathrm{T}}
$$

where $\underline{v}=Q^{-1} \underline{u}$. Apart from the ones on the diagonal, $Q^{-1} \mathrm{TQ}$ is in the form of a companion matrix for a polynomial whose coefficients are the elements of $\underline{v}$. Therefore $\underline{v}$ can be chosen so as to make the roots equal to $\left\{\lambda_{i}-1\right\}$, in which case $\underline{\underline{l}}=A^{-1} Q \underline{v}$ will cause $S$ to have the desired eigenvalues. This uniquely determines $\underline{\ell}$.

It remains to be shown that the following lemma is true.

Lemma. If $\mathrm{A}$ is an upper triangular matrix such that $A_{i i}=c$ (i not summed) and if $A_{i, i+1} \neq 0, i=0,1, \ldots, k-1$, then there exists a similarity transformation ' $Q$ with the above form that takes $A$ into a Jordan form without changing its diagonal or first upper diagonal.

Proof. By construction, let $Q_{i}$ be the elementary transformation

$$
Q_{i}=\left[\begin{array}{cccccccc}
1 & 0 & 0 & \ldots & \ldots & \ldots & 0 \\
0 & 1 & 0 & \ldots & \ldots & \ldots & 0 \\
\ldots & \ldots & \ldots & \ldots & \ldots & \ldots & \\
0 & \ldots & 1 & \mathrm{q}_{\mathrm{i}+1}, \mathrm{i}+2 & \ldots & \mathrm{q}_{\mathrm{i}+1, \mathrm{k}-1} \\
\ldots & \ldots & \ldots & \ldots & \ldots \\
0 & 0 & 0 & \ldots & \ldots & \ldots & 1
\end{array}\right]
$$

and let $A_{i+1}=Q_{i}^{-1} A_{i} Q_{i}, i=0,1, \ldots, k-3$, where $A_{0}=A$. The $q_{i+1, j}$ can be chosen to annihilate the last $k-i-2$ elements of the $i$ th row of $A_{i+1}$. Thus

$$
Q=\prod_{i=0}^{k-3} Q_{i}
$$

is the required transformation.

Since $A_{p k}$, the $(k-p) \times(k-p)$ lower principal minor of the $k \times k$ Pascal matrix $A_{0 k}$ is similar to $A_{0, k-p}$ under the diagonal transformation

$$
D_{p k}=\operatorname{diag}\left[d_{i}=\left(\begin{array}{c}
p+i \\
i
\end{array}\right)\right],
$$


the last $\mathrm{k}-\mathrm{p}$ elements $\underline{\ell}_{\mathrm{pk}}$ of $\underline{\ell}$ for $\mathrm{p}$ other than zero can be obtained from $\underline{\ell}_{0, \mathrm{k}-\mathrm{p}}$ by a linear transformation

$$
D_{p k} S_{p k} D_{p k}^{-1}=\left(I+D_{p k} \underline{l}_{p k} \underline{e}_{0}^{T} D_{p k}^{-1}\right) D_{p k} A_{p k} D_{p k}^{-1}=\left(I+D_{p k} \underline{p k}_{p k} \underline{e}_{0}^{T}\right) A_{0, k-p},
$$

so that $\underline{\ell}_{\mathrm{pk}}=\mathrm{D}_{\mathrm{pk}}^{-1} \underline{\ell}_{0, \mathrm{k}-\mathrm{p}}$.

The remaining elements of $\underline{l}$ can be chosen so as to get the highest degree possible. To do this, we must first define truncation error. The direct approach is to look at the difference between the value of $a_{n+1}$ calculated from the correct value of $\underline{a}_{n}$ and the correct value of $\underline{a}_{n}+1$. It will be seen below that a higher degree is possible if another definition is adopted. In practice other definitions mean that starting and step-changing errors are of a lower degree than truncation errors, although a difference of one may be tolerable since the contributions to the total error are then of the same order. However, the truncation error will be defined as follows:

Define the $\left(k^{\prime}-k\right)$-dimensional vector $\underline{r}$ to be the transpose of

$$
\left[h^{k} y^{(k)} / k !, \ldots, h^{k^{\prime}-1} y^{\left(k^{\prime}-1\right)} /\left(k^{\prime}-1\right) !\right] \text {. }
$$

Let $\underline{a}_{n}^{C}$ be the correct value of $\underline{a}_{n}$.at $x_{n}$ and suppose that $\underline{a}_{n}$ is given by

$$
\underline{a}_{n}=\underline{a}_{n}^{C}+E_{\underline{r}_{n}} \text {, }
$$

where $E$ is a $k x\left(k^{\prime}-k\right)$ matrix of constants. If the method calculates an $\underline{a}_{n+1}$ from this $\underline{a}_{n}$, which is related to the correct value $\underline{a}_{n+1}^{c}$ by

$$
\underline{a}_{n+1}=\underline{a}_{n+1}^{C}+E_{\underline{r}_{n+1}}+\underline{t}_{n} h^{k^{\prime}}+{ }_{n} O\left(h^{k^{\prime}+1}\right)
$$

for equations with solutions in $\mathrm{C}^{\mathrm{k}^{\prime}}$, then the truncation error is defined as $\underline{t}_{n} h^{k^{\prime}}+O\left(h^{k^{\prime}+1}\right)$ and the method has degree $k^{\prime}-1$. This means that the vector a that we normally calculate contains components of the derivatives of order $k$ and greater, and thus differs from the previously assumed values by $O\left(\mathrm{~h}^{\mathrm{k}}\right)$. It is unlikely that the user will calculate starting values with these components included, so errors from start-up of $O\left(\mathrm{~h}^{\mathrm{k}-\mathrm{p}+1}\right)$ can be expected. Also, the simple method for changing step size, namely premultiplying by a diagonal matrix, will not preserve $E$, and therefore also introduces errors of the same order. 
Let the $\left(k^{\prime}+1\right)$ Pascal matrix be partitioned as follows:

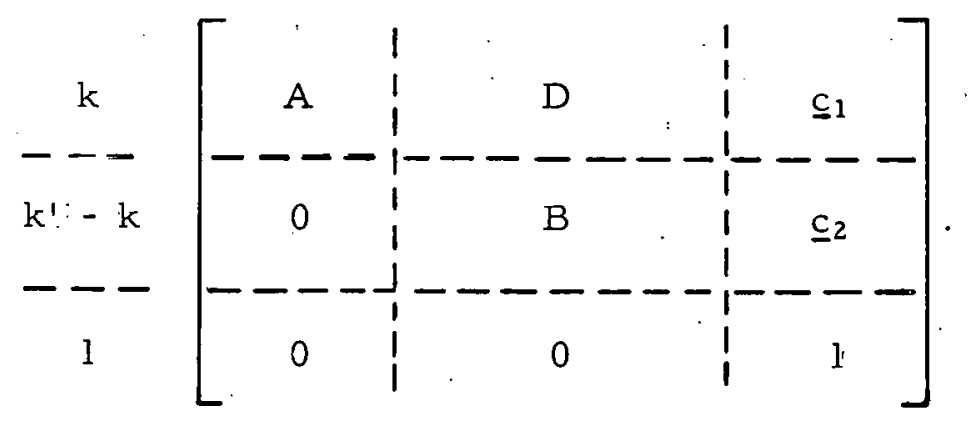

If the solution is in $\mathrm{C}^{\mathrm{k}^{\prime}+1}$, then

$$
\underline{a}_{n+i^{\prime}}^{C}=A \underline{a}_{n}^{C}+D_{\underline{r}_{n}}+\underline{c}_{1} a_{k^{\prime}}+O\left(h^{k^{\prime}+1}\right)
$$

and

$$
\underline{r}_{n+1}=B_{\underline{r}_{n}}+\underline{c}_{2} a_{k^{\prime}}+O\left(h^{k^{\prime+1}}\right)
$$

Consider one corrector iteration only so that

$$
\underline{a}_{n+1}=A \underline{a}_{n}+\underline{l} F\left(\underline{A}_{n}\right)
$$

Substitute (6.1) into (6.4) and substitute the result into (6.2) to get

$$
A\left(\underline{a}_{n}^{C}+E_{\underline{r}_{n}}\right)+\underline{\ell} F\left(A_{\underline{a}_{n}}^{C}+A E_{\underline{r}_{n}}\right)=\underline{a}_{n+1}^{C}+E_{\underline{r}_{n+1}}+\underline{t}_{n} h^{k^{\prime}}+O\left(h^{k^{\prime}+1}\right) .
$$

Substitute (6.3) into (6.5) and use $F\left(\underline{a}_{n+1}^{C}\right)=0$ to get

$$
\begin{aligned}
\underline{t}_{n} h^{k^{\prime}} & =\left[\left(I+\underline{\ell} \frac{\partial F}{\partial \underline{a}}\right)(A E-D)-E B\right] \underline{r}_{n} \\
& -\left[\underline{E} \underline{c}_{2}+\left(I+\underline{\ell} \frac{\partial F}{\partial \underline{a}}\right) \underline{c}_{1}\right] a_{k^{\prime}}+O\left(h^{k^{\prime}+1}\right) .
\end{aligned}
$$

But

$$
\frac{\partial F}{\partial \underline{a}}=\underline{e}_{p}^{T}-\sum_{q=1}^{p} \frac{y_{y}^{f}(p-q)(p-q) ! h^{q}}{p !} \underline{e}_{p-q}^{T},
$$


so that in order to satisfy $(6.6)$

$$
\begin{aligned}
& R=\left(I+\underline{\ell}_{p}^{T}\right)(A E-D)-E B=0, \\
& \underline{t}_{n}=-\left[E_{c_{2}}+\left(I+\underline{l} \underline{e}_{\mathrm{p}}^{T}\right) \underline{c}_{1}\right] y^{\left(k^{\prime}\right) /\left(k^{\prime}\right) !} \\
& +\underline{\ell} \sum_{q=1}^{p} \frac{f_{y}(p-q)(p-q) !}{p !\left(k^{\prime}-q\right) !} \underline{e}_{p-q}^{T}(A E-D) e_{k^{\prime}-k-q} y^{\left(k^{\prime}-q\right)},
\end{aligned}
$$

and

$$
\begin{aligned}
& \sum_{q=1}^{k^{\prime}-k-i-1} \frac{f_{y}(p-q)(p-q) !}{\left(k^{\prime}-q\right) !} e_{p-q}^{T}(A E-\nu) e_{k^{\prime}-k-q-i-1} y^{\left(k^{\prime}-q-i-1\right)}=0 \\
& i=0,1, \ldots, k^{\prime}-k-2 .
\end{aligned}
$$

If we write

$$
\underline{d}^{T}=\underline{e}_{p}^{T}(A E-D),
$$

and if $\ell_{k-1} \neq 0$, then (6.10) and the last k - p rows of (6.7). form a set of nonsingular linear equations for the last $k-p$ rows of $E$ and the $k^{\prime}-k$ elements of $\underline{d}$. If $d_{0}$ is nonzero, then the first $p$ rows of (6.7) form equations that can be successively solved by working up diagonals starting from the left-most, i.e.,

$$
R_{p-1,0}, R_{p-1,1}, R_{p-2,0}, R_{p-1,2}, \ldots
$$

In this process, the first $p$ elements of the last column of $E$ can be picked without restriction. In particular, if $k^{\prime}-k \leq p$, they can be picked to make the top row of $E$ zero so that at least the function value does not contain additional derivatives. This process also determines the values of $l_{i}$ for $i=$ $\mathrm{p}-\mathrm{k}^{\prime}+\mathrm{k}, \ldots, \mathrm{p}-1$; the remaining components $\ell_{\mathrm{i}}$ are determined by the values of

$$
E_{k^{\prime}-k-1, i+k^{\prime}-k}, i=0, \ldots, p-k^{\prime}+k-1 .
$$

The dependence of elements of $E$ and $\underline{l}$ on other elements can be seen from the diagram below. The element in the $(i, j)^{\text {th }}$ position is the one 
found by equating $R_{i j}=0$. These elements are treated in the order. shown by the arrows, and each time a new diagonal is started from the right column, a free parameter $E_{i, k^{\prime}-k-1}$ is introduced.

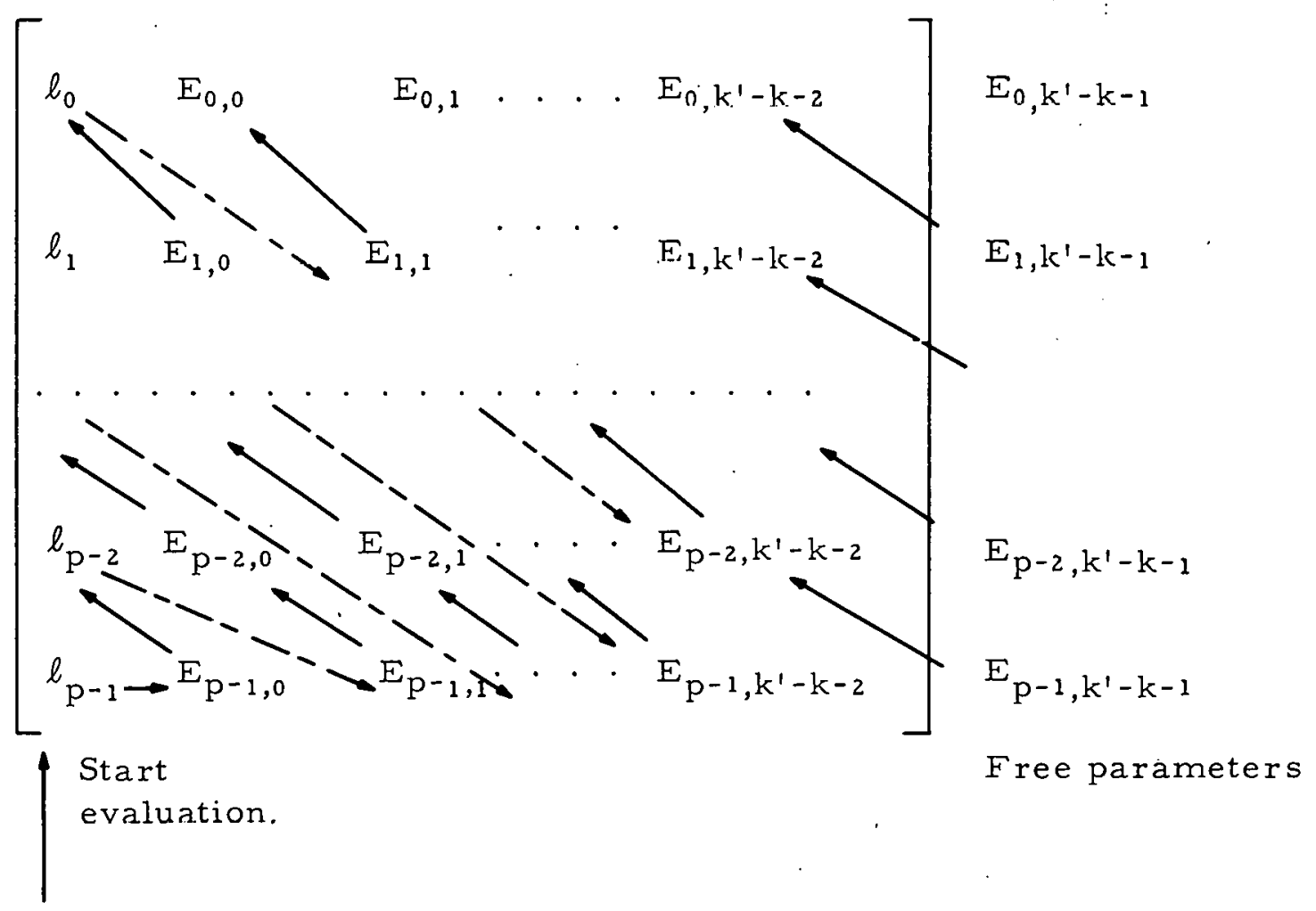

In general, (6.9) can only be satisfied by an appropriate lower-triangular part of $A E$ - D being zero or by the appropriate partial derivatives of $f$ vanishing everywhere. The former condition is independent of those parameters (the last column of $E$ ) that are still free, so that it is unlikely to be satisfied. If, however, $q$ is the smallest integer such that $\partial \mathrm{f} / \partial \mathrm{y}(\mathrm{p}-\mathrm{q}) \neq 0$, then, subject to $\mathrm{d}_{0} \neq 0$, there exists a method of degree $\mathrm{k}^{\prime}-1=\mathrm{k}+\mathrm{q}-1$. It can be seen from (6.1) and (6.7) that

$$
\mathrm{d}_{0}=\mathrm{D}_{\mathrm{k}-1,0} / \ell_{\mathrm{k}-1}=\left(\begin{array}{c}
\mathrm{k}-1 \\
\mathrm{k}-1
\end{array} / \ell_{\mathrm{k}-1}\right. \text {. }
$$

Since $l_{k-1}$ cannot be zero for a strongly stable method, $d_{0}$ exists and is nonzero. Therefore we have shown:

Theorem 2. If the smallest value of $j$ for which $\partial f / \partial y(p-j) \neq 0$ is $q$, then there exist methods of degree $k+q-l$ for all strongly stable $\underline{l}$. 


\section{CONVERGENCE AND ASYMPTOTIC ORDER OF THE ERROR}

We wish to show that sufficiently accurate stable methods will converge when applied to. Equations (5.1), and to find the asymptotic form of the error. The conditions to be used for stability will be stronger than necessary, in that multiple nonprincipal roots on the unit circle will be disallowed. Such roots may or may not make the method nonconvergent, depending on the differential equation, and are therefore of little practical importance.

Theorem 3. If a set of equations are given in the form (5.1), where the $f_{i}$ satisfy Lipschitz conditions in each variable and the solutions are sufficiently differentiable, and an initial value problem is solved for $x=0(h) b$ by means of the method

$$
\underline{a}_{i, n+1}=A_{i} \underline{a}_{i, n}+\underline{\ell}_{i} F_{i}\left(A_{j} \underline{a}_{j, n}\right) \quad i, j=1,2, \ldots, s
$$

(note that the order of each equation and of the method used for it can differ),

then, if the truncation errors in each equation as defined by (6.1) and (6.2) are bounded by $\underline{T}_{i} h^{\prime}{ }_{i}^{\prime}$ for each $i$, and if the starting errors in $\underline{a}_{i}$ are bounded by $\underline{b}_{i} h^{q_{i}}$, the numerical solution converges to the true solution and the error is asymptotically proportional to $h^{d}$, where

$$
\mathrm{d}=\min _{\mathrm{i}}\left[\mathrm{g}_{\mathrm{i}}-\mathrm{p}_{\mathrm{i}}+1, \mathrm{k}_{\mathrm{i}}^{\prime}-\mathrm{p}_{\mathrm{i}}\right] \text {, }
$$

provided that

1) The eigenvalues of each

$$
\mathrm{S}_{\mathrm{i}}=\left(\mathrm{I}-\underline{\ell}_{\mathrm{i}} \stackrel{\mathrm{e}}{\mathrm{T}}_{\mathrm{p}_{\mathrm{i}}}^{\mathrm{T}}\right) \mathrm{A}_{\mathrm{i}}
$$

are either inside the unit circle or on the unit circle and simple except for the $p_{i}$-fold root at $z=1$.

$$
\text { 2) } d \geq 1
$$

Note that the proof given below simplifies in the case $p=s=1$ to a concise proof of the usual convergence theorem given in texts.

Proof. The value of the numerical solution $\underline{a}_{i, n}$ is supposed to represent $\underline{a}_{i, n}^{C}+E_{i} \underline{x}_{i, n}$; therefore the error at $x_{n}$ will be defined as

$$
\underline{\epsilon}_{i, n}=\underline{a}_{i, n}-\underline{a}_{i, n}^{C}-E_{i} \underline{r}_{i, n}
$$


By simple manipulation, we get

$$
\begin{aligned}
\underline{E}_{i, n+1}= & A_{i} \underline{a}_{i, n}+\underline{\ell}_{i} F_{i}\left(A_{j} \underline{a}_{j, n}\right)-\underline{a}_{i, n+1}^{C}-E_{i} \underline{\underline{r}}_{i, n+1} \\
= & A_{i} \underline{\epsilon}_{i, n}+\underline{\ell}_{i} \sum_{j} \frac{\partial F_{i}}{\partial \underline{a}_{j}} A_{j} \underline{\epsilon}_{j, n}+\left[A_{i}\left(\underline{a}_{i, n}^{C}+E_{i} \underline{r}_{i, n}\right)\right. \\
& \left.+\underline{\ell}_{i} F_{i}\left(A\left(\underline{a}_{j, n}^{C}+E_{j} \underline{r}_{j, n}\right)\right)-\underline{a}_{i, n+1}^{C}-E_{i-1, n+1}\right] .
\end{aligned}
$$

By an cxtension of the definition of truncation error (6.1) and (6.2), the last term is $\underline{t}_{i, n} h^{k}$, where $\left|t_{i, n}\right| \leq T$. Noting that

$$
\frac{\partial F_{i}}{\partial \underline{a}_{j}}=\delta_{i j} \underline{e}_{p_{i}}^{T}-\sum_{q=0}^{p_{j-1}} \frac{h^{p_{i}-q} q !}{p_{i} !} \frac{\partial f_{i}}{\partial y_{j}(q)} \underline{e}_{q}^{T},
$$

we get

$\underline{\epsilon}_{i, n+1}=\left(I+\underline{\ell}_{i} \underline{e}_{p_{i}}^{T}\right) A \underline{\epsilon}_{i, n}-\underline{\ell}_{i} \sum_{j} \sum_{q} \frac{q !}{p_{i} !} h^{p_{i}-q} f_{i, y}(q) e_{j}^{T} A_{j=j, n}+\underline{\epsilon}_{i, n} h^{k !}$

Using $S_{i}=\left(I+\underline{\ell}_{i} \underline{e}_{p_{i}}^{T}\right) A_{i}$, we can express $\underline{\epsilon}_{i, N}$ as

$\underline{\epsilon}_{i, N}=S_{i}^{N} \underline{\epsilon}_{i, 0}-\sum_{m=0}^{N-1} S_{i}^{N-1-m}\left(\underline{\ell}_{i} \sum_{j} \sum_{q} k_{i, j}{ }^{p{ }^{-}-q} \underline{e}_{q}^{T} A_{j} \epsilon_{j, m}+\underline{t}_{i m}{ }^{h} k_{i}^{\prime}\right)$,

where $k_{i, j}$ are bounded parameters.

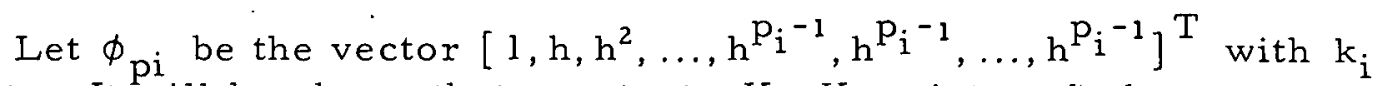
elements. It will be shown that constants $K_{1}, K_{2}$ exist such that

$$
\left|\underline{\epsilon}_{i, N}\right| \leq \Phi_{p_{i}} K_{1}\left(1+K_{2} h\right)^{N} h^{d} \leq \Phi_{p_{i}} K_{1} \exp \left(K_{2} x_{N}\right) h^{d}
$$

where $x_{N}=N h$, and $|\underline{\epsilon}|$ is the vector of absolute values of $\underline{\epsilon}$. This can best be shown by induction. For $N=0, K_{1}$ can be chosen sufficiently large that (7.4) is true for $\epsilon_{i, 0}$. 
$S_{i}$ has $p_{i}$ roots at $z=l$ and has the for $m$.

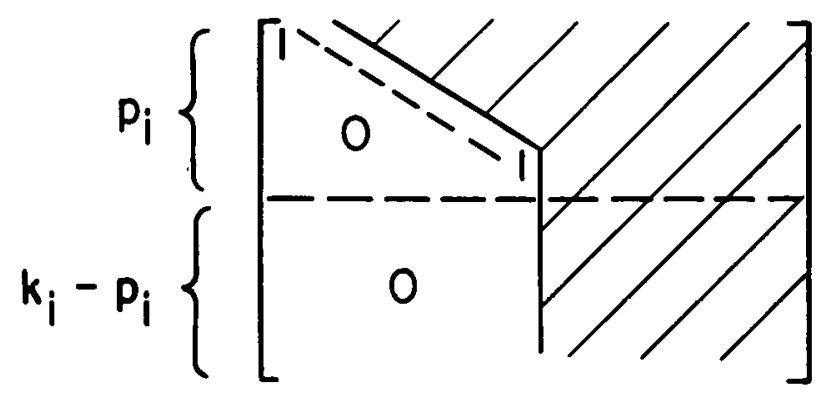

Therefore, $S_{i}^{N}$ has the following properties: The first $p_{i}$ rows of $S_{i}^{N}$ are bounded, elementwise, by $C_{1} N^{P_{i}-j-1}$ for rows $j=0,1, \ldots, p_{i}-1$. All other elements are bounded by $C_{1}$. $S_{i}^{N J}$ has the same shape as $S_{i}$. Using this bound, and assuming the truth of ${ }^{1}(7.4)$ for $\underline{\epsilon}_{i, n}, n<N$, we substitute into (7.3) to get

$$
\begin{gathered}
\left|\underline{\epsilon}_{i, N}\right| \leq h^{1-p_{i}} \underline{\phi}_{p_{i}} C_{2}\left\|\underline{\epsilon}_{0 i}\right\|+K_{1} C_{3} h^{1-p_{i}} \Phi_{p_{i}} \sum_{j} \sum_{q} h^{p_{i}-q} \underline{e}_{q}^{T} \Phi_{p_{j}} \\
\quad \sum_{m}^{i}\left(1+K_{2} h\right)^{m} h^{d}+h^{1-p_{i}} \Phi_{p_{i}} C_{4} N h^{k !} .
\end{gathered}
$$

Now

$$
\underline{e}_{q}^{\mathrm{T}} \cdot \underline{\phi}_{\mathrm{j}}=\mathrm{h}^{\mathrm{q}}
$$

and

$$
\sum_{m=0}^{N}\left(1+h K_{2}\right)^{m}=\left(\left(1+h K_{2}\right)^{N}-1\right) / h K_{2}
$$

therefore

$\left|\epsilon_{i, N}\right| \leq h^{q_{i}-p_{i}+1} C_{5} \underset{-p_{i}}{ }+\frac{K_{1}}{K_{2}} C_{6} \Phi_{p_{i}}\left(\left(1+h K_{2}\right)^{N}-1\right) h^{d}+h^{k_{i}^{\prime}-p_{i} C_{7} \Phi_{p_{i}}}$.

Since $d \leq k_{i}^{\prime}-p_{i}$ and $d \leq q_{i}-p_{i}+1$, by choosing

$$
\mathrm{K}_{1} \mathrm{C}_{6} / \mathrm{K}_{2} \geq \mathrm{C}_{5}+\mathrm{C}_{7} \text {, }
$$


we get

$$
\left|\underline{\epsilon}_{i, N}\right| \leq \frac{K_{1}}{K_{2}} C_{6} \Phi_{p_{i}}\left(1+h K_{2}\right)^{N} h^{d}
$$

and by choosing $\mathrm{K}_{2} \geq \mathrm{C}_{6}$, we have the desired result

$$
\left|\underline{\epsilon}_{i, N}\right| \leq K_{1} \Phi_{p_{i}}\left(1+h K_{2}\right)^{N} h^{d}
$$

The proof above is for the case of one corrector iteration. It can easily be extended to the general case, even if the vectors $\underline{l}$ that are used are not the same at each iteration. The stability condition is then a condition on

$$
S_{i}=\prod_{m=0}^{M-1}\left(I-\underline{\ell}_{i, m} \underline{e}_{p_{i}}^{T}\right) A_{i}
$$

With the aid of the above theorem, it is now straightforward to prove the following result about the asymptotic form of the error.

Theorem 4. If the truncation error at each step of method (7.1) applied to Equations (5.1) with solutions in $C^{\bar{k}_{i+1}}$ is written in the form

$$
\left(\ell_{i} \sum_{j} \sum_{q} \frac{\partial f_{i}}{\partial y_{j}\left(p_{i}-q\right)} y_{j}^{\left(\bar{k}_{j}-q\right)} t_{1 j q}+y^{\left(\bar{k}_{i}\right)} t_{2 i}\right) h^{\bar{k}_{i}}+o\left(h^{\bar{k}_{i+1}}\right),
$$

where $\bar{k}_{i}=d+p_{i}[\operatorname{see}(6.8)]$, and if the $t_{2 i}$ can be expressed as

$$
\underline{t}_{2 i}=\sum_{m=0}^{k_{i}-1} \alpha_{i m} s_{i}^{m} \underline{\ell}_{i}
$$

for some constants $\alpha_{i m}$, where

$$
S_{i}=\left(I+\underline{l}_{i} \underline{e}_{p_{i}}^{T}\right) A_{i}
$$

then the error in $\underline{a}_{i}$ in integrating from exact initial conditions has components due to an error $h^{d_{e}} e_{i}(x)+O\left(h^{d+1}\right)$ in the function values, where $e_{i}(x)$ is the solution from zero initial conditions of 


$$
\begin{aligned}
& e_{i}^{\left(p_{i}\right)}(x)=\sum_{q} \sum_{j} \frac{\partial f}{\partial y_{j}\left(p_{j}-q\right)}\left(e_{j}^{\left(p_{j}-q\right)}(x)+y_{j}^{\left(\bar{k}_{j}-q\right)}(x) t_{1 j q}\right)+\sum_{m} \alpha_{i m} y^{\left(\bar{k}_{i}\right)} \\
& \quad i=1,2, \ldots, s,
\end{aligned}
$$

where $\partial f / \partial y_{j}^{(s)}$ and $y_{j}^{(s)}$ are evaluated on the correct solution. The starting error can be treated similarly.

The proof is omitted since it follows the usual method for similar theorems. Condition (7.5) is annoying, since this writer has found no way to show that it can always be met, although results for first-order equations suggest that there will be no problems. It corresponds to dividing through by $\sum_{i} \beta_{i}$ in the usual multistep methods. (See Henrici, par. 5.3-5.) Since

$S_{i}$ has only one eigenvector corresponding to the eigenvalue one, almost all $\underline{\ell}$ give rise to $S$ such that $\left[S^{\mathrm{m}} \underline{\ell}: \mathrm{m}=0,1, \ldots, \mathrm{k}-1\right]$ are linearly independent, so that there are no problems in general.

\section{CONCLUSION}

Methods have been given that enable differential equations of degree greater than one to be integrated directly. Preliminary numerical evidence indicates that this process is faster and more accurate than similar methods applied to equivalent systems of first-order equations. There also appears to be some future in methods of this type for the integration of equations in the neighborhood of singularities of the family of solutions. The theoretical results for the asymptotic form of the error and for existence proofs have some holes in them of the almost-nowhere kind, but in practice they have. not affected any of the specific methods discussed, so it may well be that they will never occur. The problem of increasing the order of k-step methods beyond the Dahlquist limit for all $k$ has been laid to rest, but the problem of when to choose methods not of maximum order with other than zero nonprincipal roots remains in an enlarged form. This writer guesses that the exploitation of other spaces $\mathrm{H}_{\mathrm{k}}$ and coordinate systems for them may lead to improved methods for many problems. For example, finite Fourier series as treated by Gautschi ${ }^{5}$ would seem to be more useful for those solutions that could be thought to be band-limited, rather than having small high-order derivatives. 


\section{APPENDIX A}

Modified Predictor-Corrector Methods for First-order Equations with Nonprincipal Roots All Zero for $\mathrm{k}=2,3$, and 4

Two-step Method

Order $=4$, local error in $y, h y^{1}$ is $O\left(h^{5}\right)$.

$A=\left[\begin{array}{rrrr}-4 & 5 & 4 & 2 \\ 1 & 0 & 0 & 0 \\ -12 & 12 & 8 & 5 \\ 0 & 0 & 1 & 0\end{array}\right] ; \quad \underline{\ell}=\left[\begin{array}{c}-3 / 8 \\ 1 / 24 \\ -1 \\ 0\end{array}\right]$.

Three-step Method

Order $=6$, local error in $y$, hy' is $O\left(h^{7}\right)$.

$A=\left[\begin{array}{rrrrrr}-18 & 9 & 10 & 9 & 18 & 3 \\ 1 & 0 & 0 & 0 & 0 & 0 \\ 0 & 1 & 0 & 0 & 0 & 0 \\ -57 & 24 & 33 & 24 & 57 & 10 \\ 0 & 0 & 0 & 1 & 0 & 0 \\ 0 & 0 & 0 & 0 & 1 & 0\end{array}\right] ; \quad \underline{l}=\left[\begin{array}{l}-95 / 288 \\ +3 / 160 \\ -11 / 1440 \\ -1 \\ 0 \\ 0\end{array}\right]$.

Four-step Method

Order $=8$, local error in $y, h y^{\prime}$ is $O\left(h^{9}\right)$.

$9 A=\left[\begin{array}{rrrrrrrr}-384 & -324 & 576 & 141 & 144 & 648 & 432 & 36 \\ 9 & 0 & 0 & 0 & 0 & 0 & 0 & 0 \\ 0 & 9 & 0 & 0 & 0 & 0 & 0 & 0 \\ 0 & 0 & 9 & 0 & 0 & 0 & 0 & 0 \\ -1360 & -1350 & 2160 & 550 & 456 & 2376 & 1656 & 141 \\ 0 & 0 & 0 & 0 & y & 0 & 0 & 0 \\ 0 & 0 & 0 & 0 & 0 & 9 & 0 & 0 \\ 0 & 0 & 0 & 0 & 0 & 0 & 9 & 0\end{array}\right] ; 120960 \underline{\ell}=-\left[\begin{array}{c}36799 \\ -1375 \\ 351 \\ -191 \\ 120960 \\ 0 \\ 0 \\ 0\end{array}\right]$. 


\section{APPENDIX B}

Nordsieck's Methods (N Methods with Zero Nonprincipal

Eigenvalues) for $\mathrm{k}=2(1) 8$

The matrix $A$ is the ( $k \times k$ ) Pascal-triangle matrix. The order is $k$, and the local truncation error in $y, h y^{\prime}$ is $O\left(h^{k+1}\right)$. The corrector vectors are:

$$
\begin{aligned}
& \mathrm{k}=3: \underline{\ell}^{\mathrm{T}}=(-5 / 12 \quad-1-1 / 2) \text {, } \\
& k=4: \underline{\ell}^{\mathrm{T}}=\left(\begin{array}{lll}
-3 / 8 & -1-3 / 4 & -1 / 6
\end{array}\right) \text {, } \\
& k=5: \underline{\ell}^{T}=\left(\begin{array}{llll}
-251 / 720 & -1-11 / 12 & -1 / 3 & -1 / 24
\end{array}\right) \text {, }
\end{aligned}
$$

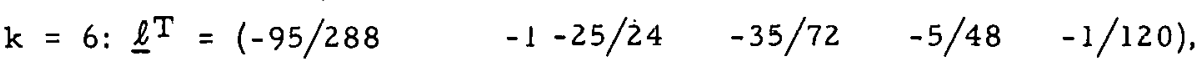

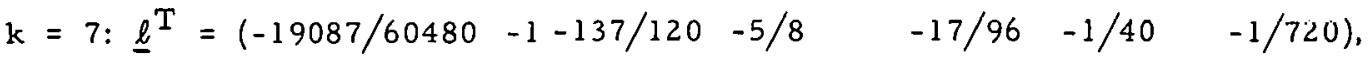

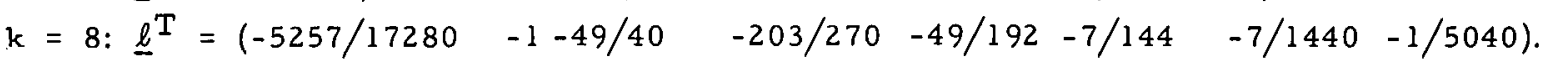




\section{APPENDIX C}

Nordsieck's Methods for $\mathrm{p}=2(1) 4$ and Various $\mathrm{k} \leq 8$ with Zero Nonprincipal Eigenvalues

The matrix $A$ is the Pascal-triangle matrix.

$\underline{p=2}$. Order of method is $k-1$; local error in $y, h y^{\prime}$, and $h^{2} y^{\prime \prime} / 2$ is $O\left(h^{k+1}\right)$.

$k=5: \underline{l}^{\mathrm{T}}=\left(\begin{array}{lllll}-19 / 90 & -3 / 4 & -1 & -1 / 2 & -1 / 12\end{array}\right)$,

$k=6: \underline{l}^{T}=\left(\begin{array}{llllll}-3 / 16 & -251 / 360 & -1 & -11 / 18 & -1 / 6 & -1 / 60\end{array}\right)$,

$k=7: \underline{\ell}^{T}=\left(\begin{array}{lllllll}-863 / 5040 & -665 / 1008 & -1 & -25 / 36 & -35 / 144 & -1 / 24 & -1 / 360\end{array}\right)$,

$k=8: \underline{\ell}^{T}=\left(\begin{array}{llllllll}-1925 / 12096 & -19087 / 30240 & -1 & -137 / 180 & -5 / 16 & -17 / 240 & -1 / 120 & -1 / 2520\end{array}\right)$.

If the first derivative is absent, then it is possible to achieve an order of one greater in $\mathrm{y}$ at the expense of introducing constant multiples of $\mathrm{y}^{(\mathrm{k})} \mathrm{h}^{\mathrm{k}}$ into $\mathrm{y}^{\prime}$ and $\mathrm{y}^{\prime \prime}$. These are equivalent to the general Cowell methods. The coefficients are identical to the case above except for $\ell_{0}$, which should be

$\mathrm{k}=5: \ell_{0}=-19 / 120$,

$\mathrm{k}=6: l_{0}=-3 / 20$,

$k=7: l_{0}=-863 / 6048$,

$\mathrm{k}=8: \ell_{0}=-275 / 2016$.

$\underline{p}=3$. Order of the method is $k-2$; local error in $y, h y^{\prime}, h^{2} y^{\prime \prime}$, and $h^{3} y^{\prime \prime \prime}$ is $O\left(h^{k+1}\right)$.

$k=5: \underline{\ell}^{T}=\left(\begin{array}{lllll}-7 / 40 & -3 / 4 & -5 / 4 & -1 & -1 / 4\end{array}\right)$

$k=6: \underline{\ell}^{T}=\left(\begin{array}{llllll}-17 / 120 & -19 / 30 & -9 / 8 & -1 & -3 / 8 & -1 / 20\end{array}\right)$,

$\mathrm{k}=7: \underline{\ell}^{\mathrm{T}}=\left(\begin{array}{lllllll}-41 / 336 & -9 / 16 & -251 / 240 & -1 & -11 / 24 & -1 / 10 & -1 / 120\end{array}\right)$,

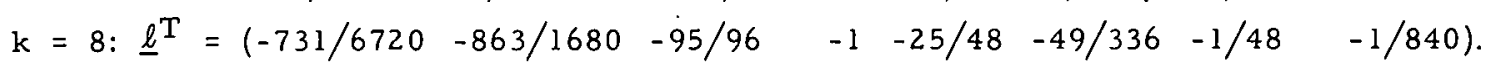

$\underline{p}=4$. Order of the method is $k-3$; local error in $y, h y^{\prime}, h^{2} y^{\prime \prime}, h^{3} y^{\prime \prime \prime}$, and $h^{4} y^{(4)}$ is $O\left(h^{k+1}\right)$.

$k=6: \underline{\ell}^{\mathrm{T}}=\left(\begin{array}{lllll}-2 / 15 & -7 / 10 & -3 / 2 & -5 / 3 & -1-1 / 5\end{array}\right)$,

$k=7: \underline{\ell}^{T}=\left(\begin{array}{llllll}-11 / 105 & -17 / 30 & -19 / 15 & -3 / 2 & -1-3 / 10 & -1 / 30\end{array}\right)$,

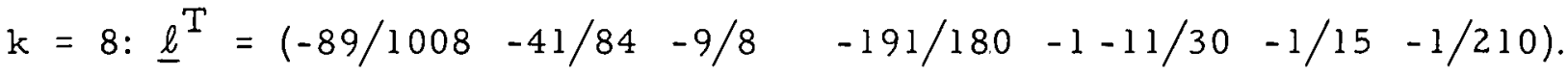




\section{REFERENCES}

1. J. C. Butcher, A Modified Multistep Method for the Numerical Integration of Ordinary Differential Equations, J. ACM 12(1), pp. 124-135 (Jan 1965).

2. Germund Dahlquist, Convergence and Stability in the Numerical Integration of Ordina ry Differential Equations, Mathematica Scandinavica 4 , pp. 33-53 (1956).

3. J. Descloux, A Note on a Paper by A. Nordsieck, Report \#131. Department of Computer Science, University of Illinois, Urbana, Ill. (Jan. 11, 1963).

4. M. Feldstein and Hans J. Stetter, Technical Report, Dept. Math., UCLA (Aug'1963).

5. W. Gautschi, Numerical Integration of Ordinary Differential Equations Based on Trigonometric Polynomials, Numerische Mathematik $\underline{3}$, pp. 381-397 (1961).

6. C. W. Gear, Hybrid Methods for Initial Value Problems in Ordinary Differential Equations. J. Soc. Indus. Appl. Math., Series B, Numerical Analysis $\underline{2}(1)$, pp. 69-86 (1965).

7. William B. Gragg and Hans J. Stetter, Generalized Multistep Predictor-Corrector Methods, J. ACM 11, pp. 188-209 (1964).

8. Peter Henrici, Discrete Variable Methods in Ordinary Differential Equations. John Wiley \& Sons, Inc., New York; N. Y. (1962).

9. Arnold Nordsieck, On Numerical Integration of Ordinary Differential Equations, Mathematics of Computation 16(77-80), pp. 22-49 (1962). 